\title{
Determinants of reaction time for digit inequality judgments
}

\author{
R. S. MOYER \\ Bates College, Lewiston, Maine 04240 \\ and \\ T. K. LANDAUER \\ Bell Laboratories, Murray Hill, N.J. 07974
}

Moyer \& Landauer (1967) found that reaction time to indicate the larger of two digits was well described by an equation proposed by Welford (1960) as a general description of decision times for inequality judgments about perceived physical magnitudes. Moyer and Landauer suggested, therefore, that perceptual and symbolic inequality judgments might be based on similar processes. Parkman (1971) presented seemingly contrary evidence, but reanalyses of his data show that they are, in fact, fully consistent with our original account.

Decisions such as "line $\mathrm{AB}$ is longer than line CD" may be termed inequality judgments. An old and quite general finding (see Welford, 1960, for review) is that inequality judgments about directly perceived physical quantities are made faster the larger the difference between the quantities. Welford (1960) summarized the usual finding as

$$
\mathrm{RT}=\mathrm{a}+\mathrm{k} \log [\mathrm{L} /(\mathrm{L}-\mathrm{S})]
$$

where $\mathrm{RT}$ is the mean reaction time, $\mathrm{L}$ and $\mathrm{S}$ the values of the larger and smaller quantities, and $\mathrm{a}$ and $\mathrm{k}$ are constants.

It might seem unlikely that digit inequality judgments, e.g., that 6 is larger than 2, should be made in the same way as those about physical stimuli, since different digits do not produce obvious ordered differences in sensation but are merely arbitrary symbols of elements in an abstract system. Nevertheless, Moyer \& Landauer (1967) found that the time required to judge which was the larger of two single digits presented visually was well described by Welford's equation. However, Parkman (1971) has suggested that our report underestimated the role of the size of the smaller digit (S) and overestimated the role of the numerical difference $(\mathrm{L}-\mathrm{S})$ between the digits. In this note we report reanalyses of our data and some of Parkman's. ${ }^{1}$ We conclude first that computational errors and statistical artifacts in Parkman's analyses led him to incorrect estimates of the relative importance of $S$ and $L-S$ and, second, that Welford's equation actually provides an even better fit to Parkman's data than to our own.

Parkman was interested in a theory of mental arithmetic which suggested that digit inequality decisions might involve counting from zero to the smaller digit. If so, RT should depend directly on S, and correlation between RT and $\mathrm{L}-\mathrm{S}$ might be due to confounding of the independent variables.

Parkman reported a two-part experiment (Experiment I). The first part (Group 1) was essentially a replication of the main experiment of Moyer \& Landauer (1967). Ss were shown all possible pairs from the set of digits $0-9$, for which $L-S$ varies between 1 and 9. In the second part (Group 2), Ss saw only the subset of digit pairs for which $\mathrm{L}-\mathrm{S}$ is 3 or less. Parkman's apparent rationale for this experiment was that if the correlation between one variable and RT remained invariant over different digit sets while the correlation between another variable and RT decreased, then the first variable could be taken to be more "fundamental."

As Columns 2 and 3 of Table 1 show, the correlation of RT with $S$ was slightly greater and that with $L-S$ somewhat less for Group 2 than for Group 1. Parkman therefore concluded that $S$ is fundamentally related to RT, while $\mathrm{L}-\mathrm{S}$ is not. However, such a conclusion is completely unwarranted. Between Groups 1 and 2 a restriction in range of $\mathrm{L}-\mathrm{S}$ was introduced, and its correlation with RT would be expected to diminish for this reason alone (see McNemar, 1962; Nunnally, 1967). We applied a standard correction for restriction of range (McNemar, 1962, p. 144) to these data. The $r$ values shown in Column 4 of Table 1 are those expected from the Group 2 design on the assumption that the observations for Group 1 (Column 2) are true population values (given the additional assumptions of linear regression and homoscedasticity). Comparison of Column 4 with Column 3 reveals that the observed

Table 1

Correlations (r) Between Reaction Time and Digit Values for Inequality Judgments

(1)

\begin{tabular}{lc} 
Variable & $\begin{array}{c}\text { Data from } \\
\text { Moyer \& } \\
\text { Landauer }^{2} \\
(1967)\end{array}$ \\
\hline Smaller Digit $(\mathrm{S})$ & .612 \\
Numerical Difference $(\mathrm{L}-\mathrm{S})$ & -.612 \\
Log $[\mathrm{L} /(\mathrm{L}-\mathrm{S})]$ & .720
\end{tabular}

(2)

Data from

Parkman (1971)

Experiment I

\begin{tabular}{cc}
\hline Group 1 & Group 2 \\
.873 & .881 \\
-.661 & -.445 \\
.912 &
\end{tabular}

(4)

Group 2

(Parkman)

Expected from Restriction Predicted by of Range $\quad \log [\mathrm{L} /(\mathrm{L}-\mathrm{S})]$

(5)
.912
0.899

$-0.691$

\begin{tabular}{rr}
.887 & 0.899 \\
-.311 & -0.691 \\
& 1.000 \\
\hline
\end{tabular}


results are close to those expected solely from the restriction in range. ${ }^{3}$

Our reanalysis of Parkman's data also uncovered two computational errors, both of which exaggerated the degree of relation between S and RT (see erratum, Journal of Experimental Psychology, 1972, 96, 183). However, even with corrected values Parkman's data indicate that $\mathrm{S}$ is more highly correlated with RT than is $\mathrm{L}-\mathrm{S}$ (although there is no such difference in the Moyer and Landauer data, see Table 1, Column 1). Parkman himself did not attempt to fit the Welford equation to his data but implied in his discussion that his estimates of the relative contributions of $S$ and $L-S$ were inconsistent with it. However, closer consideration shows otherwise. For the sets of pairs used in these experiments, $\log (\mathrm{L} / \mathrm{L}-\mathrm{S})$ is more highly correlated with $S$ than with $\mathrm{L}-\mathrm{S}$. To illustrate, we computed the expected $r$ values for $R T$ with $S$ and with $L-S$ for the set of digit pairs used in Parkman's Group 1 on the assumption that RT was a perfect linear function of $\log (\mathrm{L} / \mathrm{L}-\mathrm{S})$. As shown in Table 1 , Column 5 , the pattern of correlations reported by Parkman (Column 2) appears to be entirely consistent with Welford's equation.

Note also that $\log (\mathrm{L} / \mathrm{L}-\mathrm{S})$ is more highly correlated with RT than is $\mathrm{S}$ alone in both the Moyer and Landauer data $(\mathrm{t}=2.384$, df $=69, \mathrm{p}<.02$, two-tailed $)$ and the Parkman data, Group $1(\mathrm{t}=2.065, \mathrm{df}=87, \mathrm{p}<.04$, two-tailed). The relevant $r$ values are shown in Table 1 .

\section{CONCLUSION}

Parkman's data do not lead us to alter our proposal that RT depends on some combination of the values of both digits in the pair being judged. As an empirical description, the Welford equation fits at least as well as any alternative model considered to date. The theory suggested by this description is that judgments of symbolic quantities like digits may be made by converting them to analog "images" whose relative sizes are determined in the same way as are those of mental representations of physical stimuli. It is proposed that each of two presented digits is first converted to an imaginary line (or other percept-like entity) whose size corresponds to the numerical magnitude of the digit (Restle, 1970, has proposed a similar theory for the mental comparison of two-digit numbers). The decision as to which imaginary line (or other entity) is greater is then presumed to be made just as if the images had resulted from visual perception of actual physical stimuli.

A noteworthy virtue of such a theory is that it provides a common explanation for many kinds of inequality judgments, ranging from ones based on direct perception of quantities such as brightness to ones based on remembered properties associated with symbols like digits or object names (Moyer, 1973).

\section{REFERENCES}

McNemar, Q. Psychological statistics. (3rd ed.) New York: Wiley, 1962

Moyer, R. S. Comparing objects in memory: Evidence suggesting an internal psychophysics. Perception \& Psychophysics, 1973, in press.

Moyer, R. S., \& Landauer, T. K. Time required for judgments of numerical inequality. Nature, 1967, 215, 1519-1520.

Nunnally, J. C. Psychometric theory. New York: M cGraw-Hill, 1967.

Parkman, J. M. Temporal aspects of digit and letter inequality judgments. Journal of Experimental Psychology, 1971, 91, 191-205.

Restle, F. Speed of adding and comparing numbers. Journal of Experimental Psychology, 1970, 83, 274-278.

Sekuler, R., Rubin, E., \& Armstrong, R. Processing numerical information: A choice time analysis. Journal of Experimental Psychology, 1971, 90, 75-80.

Welford, A T. The measurement of sensory-motor performance: Survey and reappraisal of twelve years' progress. Ergonomics, $1960,3,189-230$.

\section{NOTES}

1. We thank $J$ Parkman for generously providing his data. 2. In Moyer \& Landauer (1967), pairs like 6,9 and 9,6 were combined. Here, following Parkman, they are treated separately. 3. Ss in Parkman's Group 2 had less reason to believe that any particular digit was going to be the larger or smaller, without actually comparing it with the other digit, than they did in Group 1 . The underlying similarity of results between Groups 1 Group 1. The underlying similarity of results between Groups 1 Landauer (1967), based on a control experiment, and of Sekuler, Rubin, \& Armstrong (1971) that experimental context effects do not contribute importantly in this task.

(Received for publication December 11, 1972.) 